\title{
A SINGULAR FUNCTIONAL-DIFFERENTIAL EQUATION
}

\author{
P.D. SIAFARIKAS \\ Department of Mathematics \\ University of Patras \\ Patras - GREECE \\ (Received December 3, 1981 and in revised form February 11, 1982)
}

ABSTRACT. The representation of the Hardy-Lebesque space by means of the shift operator is used to prove an existence theorem for a singular functional-differential equation which yields, as a corollary, the well known theory of Frobenius for second order differential equations.

KEY WORDS AND PHRASES. Singular functional-differential equation, Hardy-Lebesque space, Shift-operator.

1980 MATHEMTICS SUBJECT CLASSIFICATION CODES. 34K05, $47 A 67,47637$.

1. INTRODUCTION.

Consider the singular functional-differential equation

$$
z^{2} y^{\prime \prime}(z)+z p(z) y^{\prime}(z)+q(z) y(z)+\sum_{i=1}^{m} a_{i}(z) y\left(q^{i} z\right)=0, \quad|q| \leq 1
$$

where

$$
p(z)=\sum_{n=0}^{\infty} a_{n} z^{n}, \quad q(z)=\sum_{n=0}^{\infty} b_{n} z^{n} \text { and } a_{i}(z)=\sum_{j=0}^{\infty} a_{i j} z^{j}, \quad i=1,2, \ldots, m
$$

are analytic functions in some neighborhood of the closed unit disk $\bar{\Delta}=\{z<\phi:$ $|z| \leq 1\}$.

We consider the problem of finding conditions for Equation (1.1) to have solutions in the space $H_{2}(\Delta)$, i.e. the Hilbert space of functions $f(z)=\sum_{n=1}^{c} a(n) z^{n-1}$ which are analytic in the open unit disk $\Delta=\{z \in \phi:|z|<1\}$ and satisfy the con$\operatorname{dition} \sum_{n=1}^{\infty}|a(n)|^{2}<+\infty$. We shall prove the following. 
THEOREM. Let

$$
k(k-1)+a_{0} k+b_{0}=0
$$

be the idicial equation of the unperturbed equation (1.1).

(i) If $2 k+a_{0}-1=\delta=k_{1}-k_{2} \neq \pm n, n=1,2, \ldots$, then Equation (1.1) has two linearly independent solutions of the form:

$$
y_{1}(z)=z^{k_{1}} u(z) \text { and } y_{2}(z)=z^{k_{2}} u(z) \text {, }
$$

where $k_{1}$ and $k_{2}$ are the roots of Equation (1.2) and $u(z)$ belongs to $H_{2}(\Delta)$.

(ii) If $2 k+a_{0}-1=\delta=k_{1}-k_{2}=0$, 1.e. $k_{1}=k_{2}$, then Equation (1.1) has only one solution of the form:

$$
y(z)=z^{k} u(z),
$$

where $k$ is the double root of Equation (1.2) and $u(z)$ belongs to $H_{2}(\Delta)$.

(iii) If $2 k+a_{0}-1=\delta=k_{1}-k_{2}=n, n=1,2, \ldots$, then Equation (1.2) has always a solution of the form:

$$
y(z)=z^{k} u(z),
$$

where $k_{1}$ is the greatest root of Equation (1.2) and $u(z)$ belongs to $H_{2}(\Delta)$.

This theorem obviously generalizes the well known Frobenius theory [1] for the Fuchs differential equations:

$$
z^{2} y^{\prime \prime}(z)+z p(z) y^{\prime}(z)+q(z) y(z)=0,
$$

which is a particular case of Equation (1.1).

Denote an abstract separable Hflbert space over the complex field by $H$, the Hardy-Lebesque space by $H_{2}(\Delta)$, an ortho-normal basis in $H$ by $\left\{e_{n}\right\}_{n=1}^{\infty}$, and the unilateral shift operator on $H\left(V: V e_{n}=e_{n+1}\right)$ by $V$. We can easily see that the following statements hold:

(1) Every value $z$ in the unit disk $(|z|<1)$ is an eigenvalue of $V *\left(V *: V * e_{n}=e_{n-1}, n \neq 1, V * e_{1}=0\right)$, the adjoint of $v$. The eigenelements $f_{z}=\sum_{n=1}^{\infty} z^{n-1} e_{n}$ form a complete system in $H$, in the sense that if $f$ is orthogonal to $f_{z}$, for every $z:|z|<1$ then $f=0$.

(i1) The mapping $f(z)=\left(f_{z}, f\right), f \in H$ is an isomorphism from $H$ onto $H_{2}(\Delta)$. 
(iii) The diagonal operator $C_{0}: C_{0} e_{n}=n_{n}, n=1,2, \ldots$, has a self-adjoined extension in $H$ with a compact inverse $B: B_{n}=\frac{1}{n} e_{n}, n=1,2, \ldots$, Moreover, if $f(z)=\left(f_{z}, f\right)$ then

$$
\begin{aligned}
& z^{n} f(z)=\left(f_{z}, V^{n} f\right) \\
& f^{(n)}(z)=\left(f_{z},\left(C_{0} V^{*}\right)^{n_{f}}\right) \\
& z f^{\prime}(z)=\left(f_{z},\left(C_{0}-I\right) f\right) .
\end{aligned}
$$

We shall use the proposition 1 of Reference [2].

\section{PROOF OF THE THEOREM.}

The transformation $y(z)=z^{k} u(z)$, reduces Equation (1.1) in the following:

$z u^{\prime \prime}(z)+\left(h_{0}+h_{1} z+h_{2} z^{2}+\ldots\right) u^{\prime}(z)+\left(\rho_{0}+\rho_{1} z+\rho_{2} z^{2}+\ldots\right) u(z)+\sum_{i=1}^{m} q^{i k} a_{i}(z) u\left(q^{i} z\right)=0$,

where $k(k-1)+k a_{0}+b_{0}=0,2 k+a_{0}=h_{0}, a_{1}=h_{1}, a_{2}=h_{2}, a_{3}=h_{3}, \ldots$ and $k a_{1}+b_{1}=\rho_{0}$, $\mathrm{ka}_{2}+\mathrm{b}_{2}=\rho_{1}, \mathrm{ka}_{3}+\mathrm{b}_{3}=\rho_{2}, \ldots$. Following Reference [2], we define the operators $\mathrm{R}_{1}, \mathrm{R}_{2}, \ldots, \mathrm{R}_{\mathrm{m}}$ on $\mathrm{H}_{2}(\Delta)$ as

$$
R_{1} u(z)=u(q z),|q| \leq 1, R_{2} u(z)=u\left(q^{2} z\right)=R_{1}^{2}(u(z)) \ldots R_{m} u(z)=u\left(q_{m} z\right)=R_{1}^{m} u(z)
$$

Thus the operator $R$ : $R u(z)=\sum_{i=1}^{m} q^{i k} a_{i}(z) u\left(q^{i} z\right),|q| \leq 1$, on $H_{2}(\Delta)$ is represented in the space $H$ by the operator

$$
\tilde{R}: \quad \tilde{R} u=\sum_{i=1}^{m} q^{i k} a_{i}^{*}(V)\left(\tilde{R}_{1}^{*}\right)^{i} u
$$

where $\tilde{R}_{1}$ is defined on $H$ as $R_{1} e_{n}=q^{n-1} e_{n}, n=1,2, \ldots$. The equation (2.1) has a solution in $\mathrm{H}_{2}(\Delta)$ if and only if the operator equation

$$
\left[\mathrm{V}\left(\mathrm{C}_{0} \mathrm{~V} *\right)^{2}+\phi_{1}(\mathrm{~V}) \mathrm{C}_{0} \mathrm{~V} *+\phi_{2}(\mathrm{~V})+\tilde{\mathrm{R}}\right] \mathrm{u}=0
$$

has a solution $u$ in the abstract separable Hilbert space $H$.

$$
\begin{gathered}
\text { Here } u=\sum_{n=1}^{\infty}\left(\overline{u, e_{n}}\right) e_{n}, \phi_{1}(v)=\left(2 k+a_{0}\right) I+h_{1} v+h_{2} v^{2}+\ldots, \\
\phi_{2}(v)=\rho_{0} I+\rho_{1} v+\rho_{2} v^{2}+\ldots,
\end{gathered}
$$

where the bar denotes complex conjugation.

Taking into account the relations

$$
\mathrm{v}^{2} \mathrm{C}_{0} \mathrm{~V} *=\mathrm{V}\left(\mathrm{C}_{0}-\mathrm{I}\right) \text { and } \quad \mathrm{VC}_{0}-\mathrm{C}_{0}=-\mathrm{V} \text {, }
$$

Equation (2.2) can be written as 


$$
\left[\left[C_{0}+\left(2 k+a_{0}-1\right) I+B \phi(V)-B^{2} V \phi_{1}^{\prime}(V)\right] V^{*}+B \phi_{2}(V)+B \overline{\tilde{R}}\right] u=0,
$$

where

$$
\phi(\mathrm{v})=\mathrm{h}_{1} \mathrm{v}+\mathrm{h}_{2} \mathrm{v}^{2}+\mathrm{h}_{3} \mathrm{v}^{3}+\ldots \text { and } \phi_{1}^{\prime}(\mathrm{v})=\mathrm{h}_{1}+2 \mathrm{~h}_{2} \mathrm{v}+3 \mathrm{~h}_{3} \mathrm{v}^{2}+\ldots .
$$

Also, if we put $2 k+a_{0}-1=\delta$ in Equation (2.3), we have

$$
\mathrm{V} *[\mathrm{I}+\mathrm{VK}] \mathrm{u}=0
$$

where the operator

$$
\mathrm{K}=\delta \mathrm{BV} *+\mathrm{B}^{2} \phi(V) \mathrm{C}_{0} \mathrm{~V} *+\mathrm{B}^{2} \phi_{2}(\mathrm{~V})+\mathrm{B}^{2} \widetilde{\mathrm{R}}
$$

is compact. Relation (2.4) implies that

$$
(I+V K) u=c e_{1}, \quad c=\text { const. }
$$

Now it follows that the operator $(\mathrm{I}+\mathrm{VK})^{-1}$ exists. In fact,

$$
(I+V K) u=0 \Rightarrow u=-V K u=>\left(u, e_{1}\right)=-\left(K u, V * e_{1}\right)=0 \text {. Also, }
$$

$$
\left(u, e_{2}\right)=-\left(u, K * e_{1}\right) \Rightarrow\left(u, e_{2}\right)(1+\delta)=0
$$

Relation (2.6) if $\delta \neq-1 \Rightarrow\left(u, e_{2}\right)=0$. Similarly,

$$
\left(u, e_{3}\right)=-\left(u k^{*} e_{2}\right) \Rightarrow\left(u, e_{3}\right)\left(1+\frac{\delta}{2}\right)=0 .
$$

Relation (2.7) if $\delta \neq-2 \Rightarrow\left(u, e_{3}\right)=0$. By the same way and if $\delta \neq-n, n=1,2, \ldots$, we find

$$
\mathrm{u}=\sum_{\mathrm{n}=1}^{\infty}\left(\overline{\mathrm{u}, \mathrm{e}_{\mathrm{n}}}\right) \mathrm{e}_{\mathrm{n}}=0 .
$$

Since also the operator VK is compact Fredholm alternative implies that the operator $(I+V K)^{-1}$ is defined every where. Thus from Equation (2.5), we have

$$
u=c \cdot(I+V K)^{-1} e_{1} \text {. }
$$

This means that

(i) If $2 \mathrm{k}+\mathrm{a}_{0}-1=\delta=\mathrm{k}_{1}-\mathrm{k}_{2} \neq \pm \mathrm{n}$ with $\mathrm{n}=1,2, \ldots$, then the operator $(I+V K)^{-1}$ always exists. Therefore, Equation (1.1) has two linearly independent solutions of the form

$$
\mathrm{y}_{1}(\mathrm{z})=\mathrm{z}^{\mathrm{k}_{1}} \mathrm{u}(\mathrm{z}) \text { and } \mathrm{y}_{2}(\mathrm{z})=\mathrm{z}^{\mathrm{k}_{2}} \mathrm{u}(\mathrm{z}),
$$

where $k_{1}$ and $k_{2}$ are the roots of Equation (1.2) and $u(z)$ belongs to $H_{2}(\Delta)$ and is given by the relation

$$
u(z)=\left(u_{z}, u\right), u_{z}=\sum_{n=1}^{\infty} z^{n-1} e_{n}, u=c \cdot(I+V K)^{-1} e_{1} .
$$


(ii) If $2 k+a_{0}-1=\delta=k_{1}-k_{2}=0$, i.e. $k_{1}=k_{2}$, then the operator $(I+V K)^{-1}$ always exists. Therefore, Equation (1.1) has only one solution of the form

$$
y(z)=z^{k} u(z)
$$

where $\mathrm{k}$ is the double root of Equation (2.1) and $\mathrm{u}(\mathrm{z})$ as in (i).

(iii) If $2 k+a_{0}-1=\delta=k_{1}-k_{2}=n, n=1,2, \ldots$, then

$$
\begin{aligned}
& 2 \mathrm{k}_{1}+\mathrm{a}_{0}-1=\mathrm{n}, \mathrm{n}=1,2, \ldots, \\
& 2 \mathrm{k}_{2}+\mathrm{a}_{0}-1=-\mathrm{n}, \mathrm{n}=1,2, \ldots,
\end{aligned}
$$

From the above and the Relations (2.6) and (2.7), we see that Equation (1.1) has always a solution of the form

$$
y(z)=z^{k_{1}} u(z),
$$

where $k_{1}$ is the greatest root af Equation (1.2) and $u(z)$ as in (i). A11 the above complete the proof of the theorem.

ACKNOWLEDGEMENTS. I am grateful to Professor E.K. Ifantis, for suggesting the topic of this research and for his continuous interest.

\section{REFERENCES}

1. HILlE, E. "Ordinary differential equation in the complex domain", WileyInterscience, 1976.

2. IFANTIS, E.K. An Existence theory for functional-differential equations and functional differential systems, Jour. Diff. Equat. 29, No. 1 (1978), 86-104. 


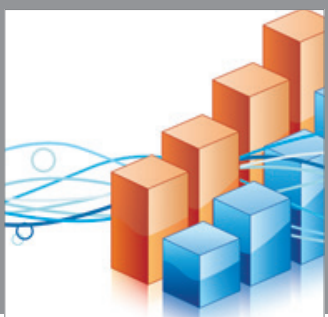

Advances in

Operations Research

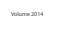

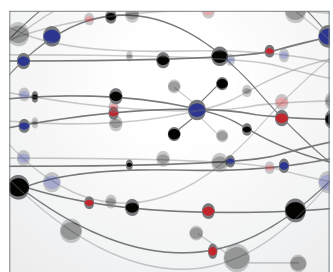

\section{The Scientific} World Journal
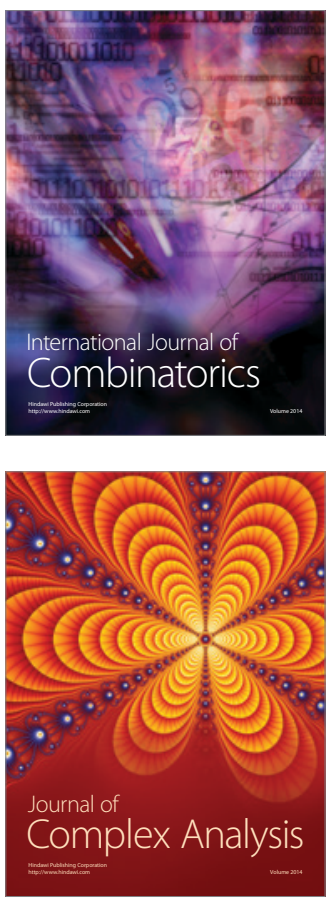

International Journal of

Mathematics and

Mathematical

Sciences
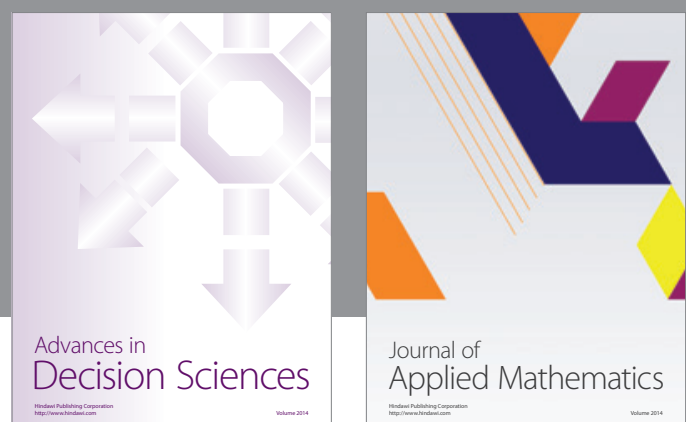

Journal of

Applied Mathematics
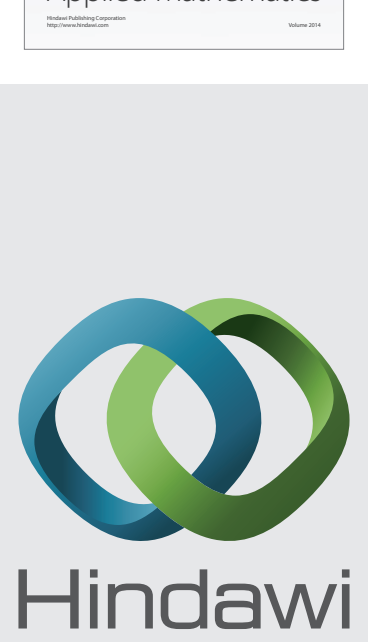

Submit your manuscripts at http://www.hindawi.com
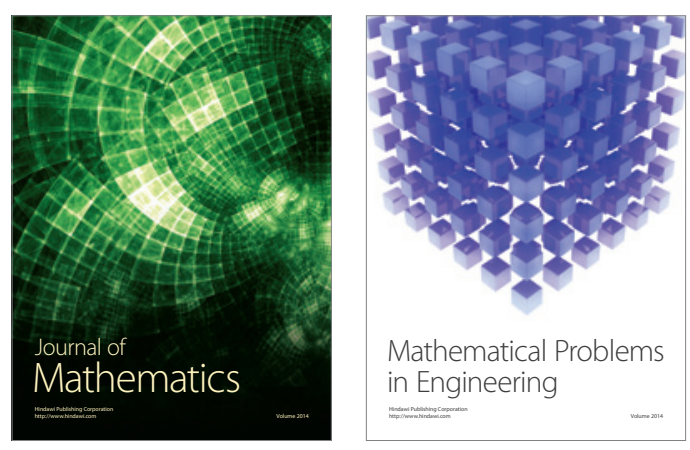

Mathematical Problems in Engineering
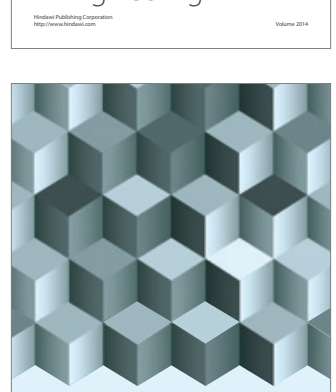

Journal of

Function Spaces
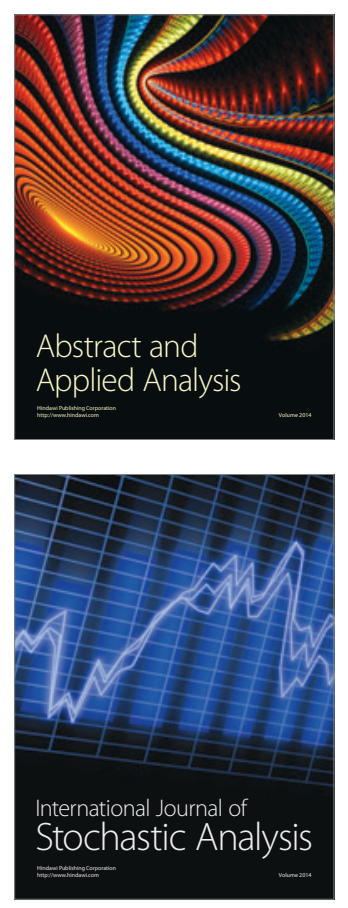

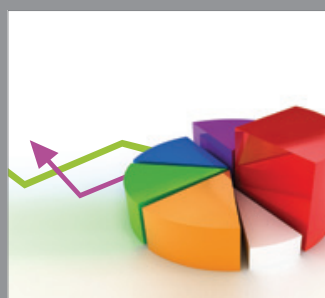

ournal of

Probability and Statistics

Promensencen
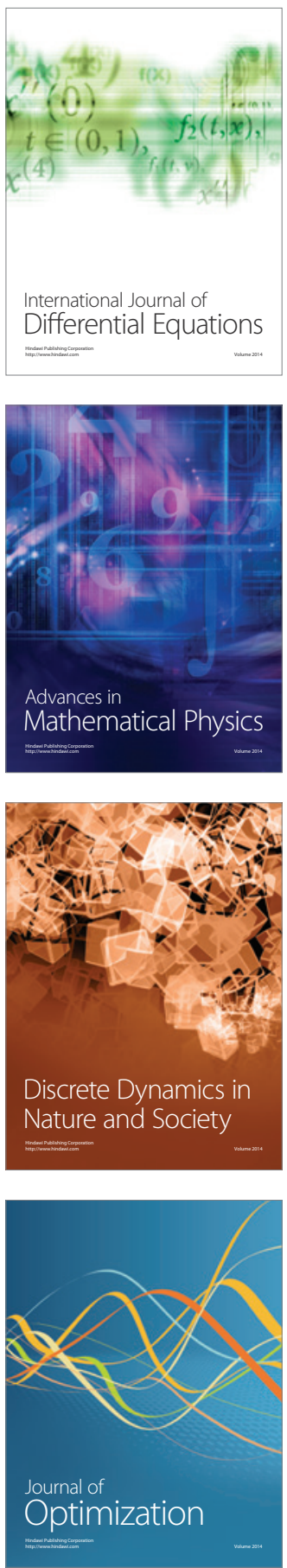